\title{
An Undiagnosed Case of Epilepsy for 13 Years Presenting in Status Epilepticus
}

\author{
Bhabani Sankar Dhal ${ }^{1}$, Anupam Duttaㄹ ${ }^{2}$ Ajit Kumar Pegu ${ }^{3}$, Srimanta Madhab Baruah ${ }^{4}$, Chayanika Dutta ${ }^{5}$
}

${ }^{1}$ Department of Medicine, Assam Medical College and Hospital, Dibrugarh, Assam, India. ${ }^{2}$ Department of Medicine, Assam Medical College and Hospital, Dibrugarh, Assam, India. ${ }^{3}$ Department of Medicine, Assam Medical College and Hospital, Dibrugarh, Assam, India. ${ }^{4}$ Department of Medicine, Assam Medical College and Hospital, Dibrugarh, Assam, India. ${ }^{5}$ Department of Medicine, Assam Medical College and Hospital, Dibrugarh, Assam, India.

\section{INTRODUCTION}

Epilepsy, one of the most common neurological diseases globally is a chronic noncommunicable disease that affects people of all ages. Around 50 million people worldwide are suffering from epilepsy at present. The risk of premature death is up to three times higher than that of general population. Up to $70 \%$ of the people living with epilepsy could live seizure- free if properly diagnosed and treated.[1] General examination is more often missed in an epilepsy patient or seizure disorder patient, but unfortunately it plays an important role when diagnosis is concerned. Here we mention a case report of 14 year old girl suffering from epilepsy, who remains undiagnosed for 13 years with characteristics clinical and radiological features of Tuberous sclerosis.

Tuberous sclerosis is a type of true phakomatoses (congenital neuroectodermoses). As the name suggests it mainly affects the CNS and cutaneous system. It is characterised by development of benign tumours affecting brain, skin, retina, visceras and cutaneous changes. It is an autosomal dominant disorder but with variable penetrance in which a variety of lesions in the skin, kidney, heart, nervous system and other organs. Bourneville's articles (between 1880-1900), presented the first systematic features of the disease and related the cerebral lesions to those of skin of the face. It is equally frequent in all the races and in both the sexes.[2] The incidence is estimated to be 1 case per 6000 live birth, with a prevalence of 1 in 10,000 births.[3] The mutation occurs either in one of the two sites-the long arm of chromosome known as TSC 1 (Hamartin), or in the short arm of chromosome 16 known as TSC 2 (tuberin). Because of mosaicism approximately 15\% of sporadic cases show no identifiable mutation and tend to have milder manifestations. In a study conducted on 325 TSC individuals, $17 \%$ of the mutations were found in the TSC1 gene, $50 \%$ in the TSC2 gene, $4 \%$ unclassified variants, and $29 \%$ with no identifiable mutations. ${ }^{[4]}$ The disease is basically a triad of epilepsy, developmental delay and adenoma sebaceum known as "EPILOIA".[2] Here we present a case report of 14 year old female patient with clinical and radiological features of TSC.
Corresponding Author: Dr. Bhabani Sankar Dhal, $2^{\text {nd }}$ Year PGT, Department of Medicine, Assam Medical College, Dibrugarh, Assam, India. E-mail: iambhabs11@gmail.com

DOI: $10.14260 /$ jemds/2020/151

Financial or Other Competing Interests: None.

How to Cite This Article:

Dhal BS, Dutta A, Pegu AK, et al. An undiagnosed case of epilepsy for 13 years presenting in status epilepticus. J. Evolution Med. Dent. Sci. 2020;9(09):697-699, DOI: 10.14260/jemds/2020/151

Submission 26-12-2019,

Peer Review 04-02-2020,

Acceptance 11-02-2020,

Published 02-03-2020.

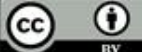




\section{PRESENTATION OF CASE}

A 14-year-old female patient presented with multiple episodes of convulsions which were generalised tonic clonic in nature and there is complete loss of consciousness in between episodes. She was a known epileptic since 4 months age and was on medication previously but discontinued it. Mother had given the history of various type of seizures (clonic, partial, myoclonic) previously and intellectual disability and regression of growth milestones. She could not talk, understand, sense her bowel and bladder habits at the appropriate age, she never went to school. None of the family members has ever suffered from similar illness.

On general physical examination, multiple well defined, brownish sessile nodular growths were noted over the forehead, nasal folds, nose and cheeks in a "butterfly pattern" suggestive of adenoma sebaceum [figure 1]. Over lumbo-sacral area multiple raised hypermelanotic skin patches were noted characteristically suggestive of shagreen patch [figures 2, 3] Multiple hypomelanotic patches with characteristically fish tailing appearance were found over median part of right thigh, abdomen, and largest one of approximately $5 \times 5 \mathrm{~cm}^{2}$ over right buttock [figures 4-7]. Vitals signs were found to be within satisfactory limits.
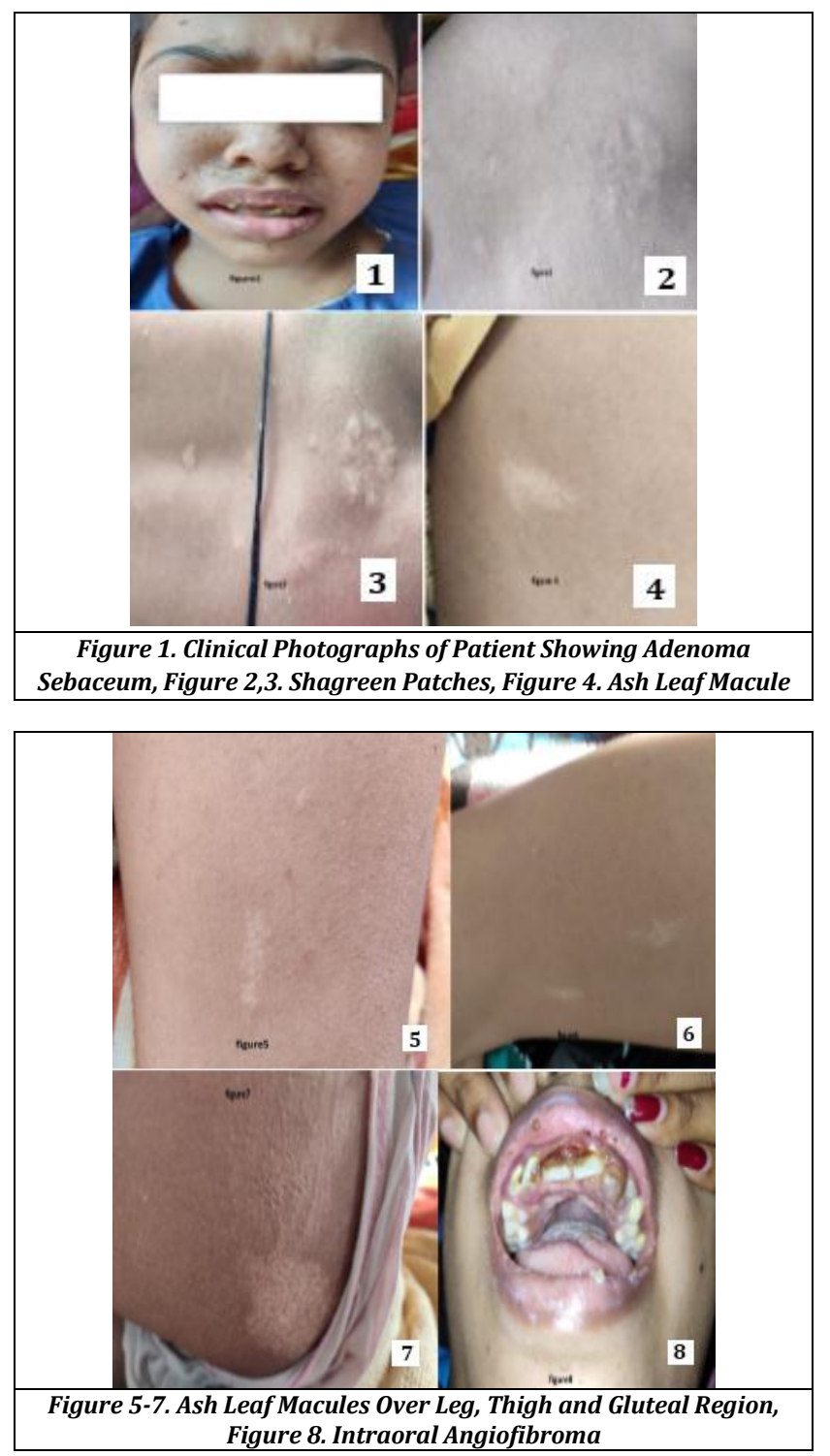

On intraoral examination, similar well defined, sessile, firm, nodular growths were seen in the attached gingiva in the upper anterior gingiva of various sizes [figure 8]. Based on history and clinical findings drug induced gingival hyperplasia ruled out.

The patient was subjected to various radiological, haematological investigations. Axial computed tomographic sections of the brain showed multiple calcified subependymal nodules in subependymal regions of both ventricles [figure 9]. Ultrasound of whole abdomen reveal multiple calcified lesions in both the kidneys [figure 10] and patient was advised for CT abdomen in the next follow up. Ophthalmology consultation was given, retina was normal, there was no signs of phakoma. Echocardiography shows no signs of rhabdomyoma. Dermatology consultation was taken to confirm the tuberous sclerosis complex.
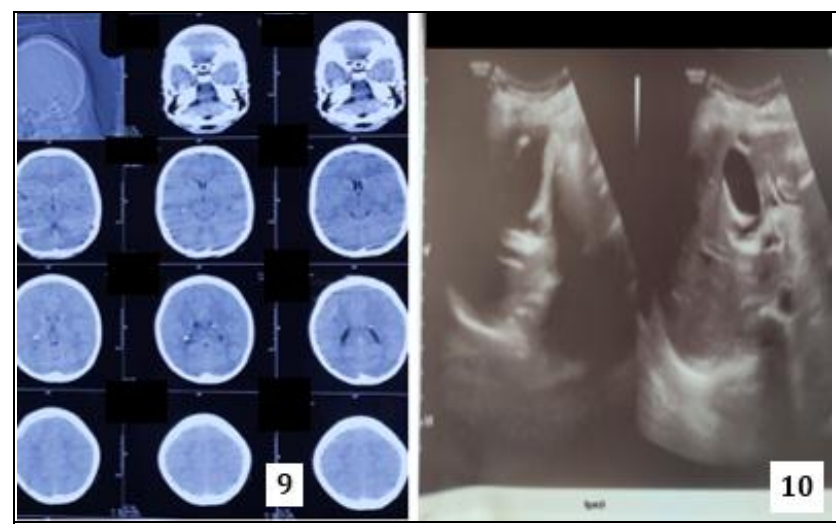

Figure 9. CT Brain Showing Multiple Subependymal Calcified Periventricular Nodules, Figure 10. USG Suggestive of Multiple Calcified Lesions in Kidney

Based on all clinical findings and investigations, a final diagnosis of tuberous sclerosis was confirmed.

\section{DISCUSSION}

The TSC gene products form a tumour suppressor complex which drives RHEB (ras homolog enriched in brain) a member of Ras superfamily into the inactive guanosine diphosphate bound state, it stimulates the Mtor (mammalian target of rapamycin), a major effector of cell growth. The mutations in these genes result in constitutive mTOR activation leading to the formation of various growths and hamartomas in various organs of the body.[5]

Most often the infant is first thought to be normal. Attention is drawn to the disease when focal or generalised seizures or by regression of milestones. The convulsive disorder and developmental delay become more prominent within 2-3 years. The cutaneous abnormality in face (adenoma sebaceum), appears later in childhood, usually between the fourth and tenth years, and progresses thereafter. In the first year the seizure is usually massive flexion spasm with hypsarrhythmia, later it changes to generalized tonic clonic or atypical petit mal type. In about $50 \%$ of the cases, grey or yellow plaques (gliomatous tumours) may be found in the retina in or the optic disc called as phakoma, term coined by Van der Hoeve. In heart benign rhabdomyomas are found, usually located in the wall of atrium, can cause conduction defect. Other benign tumours of mixed cell types (angiomyolipomas) found in the liver, lungs, kidneys, thyroid, 
testes and gastrointestinal tract. ${ }^{[2]}$

According to the Tuberous sclerosis consensus conference of 1998 two major features or one major and two minor features was considered sufficient for a definitive diagnosis. The major features include (a) hypomelanotic macules $(\geq 3$, at least $5 \mathrm{~mm}$ diameter), (b) angiofibromas ( $\geq 3$ ) or fibrous cephalic plaques, (c) shagreen patches, and (d) ungual fibromas $(\geq 2)$. The minor features include (1) intraoral fibromas $(\geq 2)(2)$ " confetti "skin lesions, and (3) dental enamel pits (z3).[6] In approximately $90 \%$ of patients of patients with tuberous sclerosis, "ash leaf" lesions (congenital hypomelanotic macules) are found. These lesions appear before the appearance of other characteristic skin lesions. In $90 \%$ of patients older than 4 years of age Adenoma sebaceum, pathognomonic of tuberous sclerosis are present. The diagnostic lesion "shagreen patch" found most often in the lumbosacral region. In the nail bed subungual fibromas usually appear at puberty and continue to develop with age. Other common skin changes include cafe-au-lait spots and port-wine haemangiomas.[2]

In the brain the walls of the lateral ventricles are encrusted with whitish masses resembling candle gutterings. When calcified, they appear in CT scan as sub ependymal calcified nodules. Neoplastic transformation of abnormal glial cells usually takes the form of a large cell astrocytoma (SEGA). Histology from the tumour like growths in different organs may include cells of more than one type (fibroblasts, cardiac myoblasts, angioblasts, glioblasts, and neuroblasts etc.). Serial examinations to detect enlargement of the subependymal tumours is advised annually for those younger than age 21 subependymal nodules, shagreen patch and hypomelanotic macules and one minor feature -gingival fibroma.[2]

The management of these patients often involves the neurosurgeon, neurologist, nephrologist, pulmonologist, cardiologist, ophthalmologist, and the genetic counsellor. Surgery including dermabrasion and laser treatment may be useful for the treatment of skin lesions. Antiepileptics are the mainstay of treatment.[7] Excision of tumours is usually pointless, especially in the severely affected individuals except the renal hamartomas, that may impair renal function. However, Sirolimus, everolimus which suppresses the mTOR signalling pathway can reduce the tumour sizes and decrease the frequency of seizures. According to recent trials use of topical $0.1 \%$ rapamycin on facial angiofibromas is beneficial. ${ }^{[2,8,9]}$ In the present case, the patient was advised to take neurology consultation for the starting of sirolimus/everolimus. At present she is taking phenytoin 300 $\mathrm{mg}$ and carbamazepine $200 \mathrm{mg}$. approximately 30\% of patients die before the fifth year, and $50-75 \%$ of patients before attaining adult age. However, in the case of individual diagnosed late in the life with few cutaneous signs, prognosis depends on the associated internal tumours and cerebral calcifications.[2]

\section{ACKNOWLEDGEMENT}

We would like to thank Dr. Purnabrat Kashyap, Dr. Trinayani Barua, Dr. Kaushik Kakoti, Dr. Bharat H.R., Dr. Balaji D., Dr. Ruth Khawbung, Dr. V.S. Visvesvaran, Dr. Azharul H. Choudhury, from the Department of General Medicine- Unit 5, Assam Medical College and Hospital for their help during the study.

\section{REFERENCES}

[1] Epilepsy- World Health Organisation. Accessed on 20 Jun $2019 . \quad$ https://www.who.int/news-room/factsheets/detail/epilepsy

[2] Ropper AH, Samuel MA, Klein JP. Adams and Victor's Principles of Neurology. 10 th edn. [eBook]. McGraw-Hill Education 2014.

[3] Schwartz RA. Genetics of Tuberous sclerosis 2019 [updated 2019 Sep 16]. Overview. https://emedicine.medscape.com/article/951002-

[4] Au KS, Williams AT, Roach ES, et al. Genotype/phenotype correlation in 325 individuals referred for a diagnosis of tuberous sclerosis complex in the United States. Genet Med 2007;9(2):88-100.

[5] Cheng TS. Tuberous sclerosis complex: an update. Hong Kong J Dermatol Venereol 2012;20:61-7.

[6] Northrup H, Krueger DA, International Tuberous Sclerosis Complex Consensus Group. Tuberous sclerosis complex diagnostic criteria update: recommendations of the 2012 international tuberous sclerosis complex consensus conference. Pediatr Neurol 2013;49(4):24354.

[7] Jankar AN, Palange PB, Purandare VC. Tuberous sclerosis - a case report. Int J Biomed Res 2014;5(10):649-50.

[8] Jameson JL, Kasper DL, Longo DL, et al. Harrison's Principles of Internal Medicine. Chap - 86. Primary and metastatic tumors of the nervous system. $20^{\text {th }}$ edn. McGraw-Hill Education 2018: p. 649. 